\title{
Stacked Integrated Double-disks for Cavity Optomechanics
}

\author{
Qiang Lin, Xiaoshun Jiang, Matt Eichenfield, Ryan \\ Camacho, Kerry Vahala, and Oskar Painter \\ California Institute of Technology, Pasadena, California 91125, USA
}

The coupling of mechanical oscillators and optical cavity modes through scattering forces has received considerable attention in recent years [1]. This interaction provides a way, through the principle of dynamic back action [2], to amplify [2,3] and cool mechanical motion [4-6]. It could also soon provide a practical means to entangle macroscopic mechanical motion with a variety of other quantum systems, including light [7,8]. To date, experimental work has relied upon the optical scattering force to create conditions necessary for observation of dynamical back action effects. However, alongside the scattering force there are also dipole optical forces that can furnish optomechanical coupling. These forces, also referred to as dispersive or gradient forces, have been used to control coupling of a waveguide to a resonator [9] and to couple pairs of waveguides $[10,11]$. In the present work, a stacked, double-disk whispering gallery system is demonstrated as a new means to cavity optomechanical phenomena. Dipole-force coupling between the disks creates optomechnical coupling, causing displacement of the disks and tuning of the underlying whispering gallery resonances. In comparison to scattering-force-based systems, this double-disk configuration has the significant advantage of providing a larger optomechanical coupling constant, independent of the cavity round trip length.

The geometry is illustrated in Fig. 1 and features a pair of whispering-gallery microcavities separated by a thin gap region. Due to their close proximity, the modes of each resonator will couple by way of their respective evanescent fields, which penetrate the gap region. From a coupled-mode viewpoint, the resulting modes of the system are symmetric and anti-symmetric combinations of the original, uncoupled, whisperinggallery modes. The electromagnetic energy associated with the combined system is strongly dependent upon the separation between the disks, creating a mutual coupling force that is repulsive for excitation of the antisymmetric mode (anti-bonding mode) and attractive for the symmetric mode (bonding mode). The coupling of optical power into either one of these modes will therefore induce a change in the gap distance. This change, in turn, tunes the spectrum of modes of the coupled system. When a pump wave is properly detuned from resonance, dynamical back action effects become observable. In the present work, strong regenerative oscillations are observed with a characteristic threshold. Beyond its use as a vehicle for study of dynamic back action, this system can also provide an optically controlled filter (when the mechanical Q factor is spoiled). Prospects for design of widely tunable filter functions will be discussed.

(a)

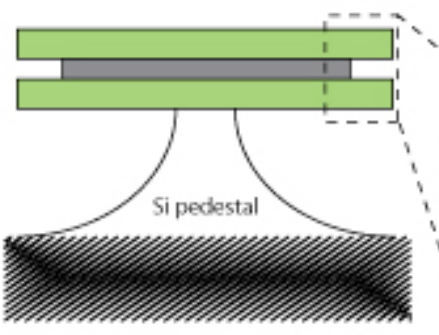

(b)

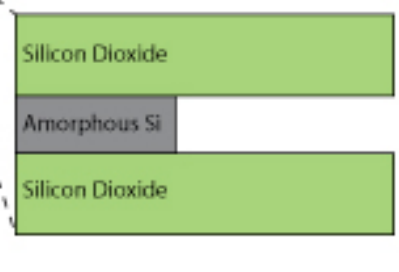

(c)

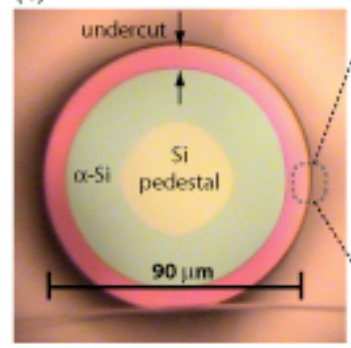

(d)

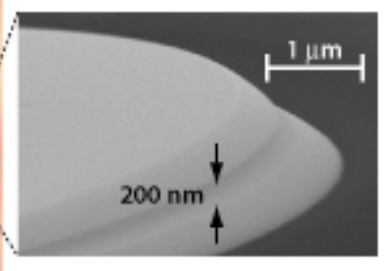

Fig. 1 (a) Schematic of a double-disk resonator consisting of a pair of high-Q silica whispering gallery devices that couple evanescently through the gap region. (b) Close up rendering of the dashed box (see left panel) showing the composition of the individual layers. (c) Optical micrograph of a fiber-taper-coupled double-disk microcavity system. (d) Scanning electron micrograph of the region indicated by the dashed box in (c).

\section{References}

[1] T.J. Kippenberg and K. J. Vahala, Science 321, 1172 (2008).

[2] V. B. Braginsky, S. P. Vyatchanin, Phys. Lett. A, 293, 228 (2002).

[3] T. J. Kippenberg, et. al. Phys. Rev. Lett. 95, 033901, (2005).

[4] T. Carmon, et. al., Phys. Rev. Lett., 94, 223902, (2005).

[5] S. Gigan, H.R. et. al., Nature (London) 444, 67 (2006)

[6] O. Arcizet, et. al., Nature (London) 444, 71 (2006).

[7] A. Schliesser, et. al. Phys. Rev. Lett., 97, 243905, Dec 15, (2006).

[8] S. Mancini et. al., Phys. Rev Lett., 88, no. 12, 120401-1 (2002).

[9] Marshall, W. et. al. Phys. Rev. Lett., 91, 130401 (2003).

[10] M. Eichenfeld, C. P. Michael, R. Perahia, O. Painter, Nature Photonics 1, 416 (2007).

[11] M. L. Povinelli et al., Optics Express 13, 8286 (2005).

[12] Mo Li , W. Pernice, C. Xiong, T. Baehr-Jones, M. Hochberg, H. Tang, Nature, 456, 480 (2008). 